\title{
Silicone Oil Droplets in the Schlemm's Canal: A Surprise during Prolene Hemi-gonioscopy-assisted Transluminal Trabeculotomy (Hemi-GATT)
}

\author{
Zeynep Aktas ${ }^{1}$, Ceyda Erişti Bölük ${ }^{2}$, Gokhan Gurelik ${ }^{3}$
}

\begin{abstract}
Aim and objective: To report a case with emulsified silicone oil (SO) droplets in the Schlemm's canal (SC), encountered during prolene hemigonioscopy-assisted transluminal trabeculotomy (hemi-GATT).

Background: In cases with a history of previous pars plana vitrectomy (PPV) with SO tamponade and then SO removal; glaucoma might occur secondary to the presence of emulsified SO droplets. Minimal invasive glaucoma surgeries targeting trabecular meshwork such as GATT surgery might be an ideal treatment option in these cases.

Case description: A 57-year-old man presented with uncontrolled intraocular pressure (IOP) and topical anti-glaucoma drug intolerance in his left eye. The patient had a history of PPV with SO tamponade and then intravitreal SO removal 5 years ago. He had a history of Ahmed glaucoma valve (AGV) implantation 2 years ago for the treatment of secondary glaucoma but he was still using topical anti-glaucoma medication. However, IOP was measured as 17 in the left eye. Hemi-gonioscopy-assisted transluminal trabeculotomy was performed successfully to get the patient off medication. During the surgery, SC was full of SO droplets.

Conclusion: The presence of SO droplets in the SC might contribute to the development of secondary glaucoma after PPV and SO removal. This might be another surgical indication for GATT surgery in these risky eyes.

Keywords: Gonioscopy-assisted transluminal trabeculotomy, Schlemm's canal, Silicone oil droplets.

Journal of Current Glaucoma Practice (2021): 10.5005/jp-journals-10078-1297
\end{abstract}

\section{BACKGROUND}

Secondary glaucoma after pars plana vitrectomy (PPV) might occur even after the uneventful surgeries and these cases are often refractory to all conventional treatment methods. ${ }^{1,2}$ Number of mechanisms were reported to cause secondary glaucoma but silicone oil (SO) retention is one of the most common causes of the secondary glaucomas encountered after the PPV. Emulsified SO droplets contribute to the obstruction of natural aqueous outflow pathways leading to secondary glaucoma. These SO droplets might still cause refractory secondary glaucoma even after the SO removal.

Different treatment options for this type of glaucoma are possible such as selective laser trabeculoplasty or trabeculotomy, tube surgery, diode lasers, and express shunt. ${ }^{3-5}$ However, in cases with a history of vitreoretinal surgery, gonioscopy-assisted transluminal trabeculotomy (GATT) can be an alternative treatment option if the gonioscopic examination reveals an open angle without any broad peripheral anterior synechias. ${ }^{6}$ It can also be performed after the SO removal if the patient has secondary glaucoma due to residual SO droplets in the eye.

With this case, we aimed to demonstrate the presence of SO droplets in the Schlemm's canal (SC) during the hemi-gonioscopyassisted transluminal trabeculotomy (hemi-GATT) surgery.

\section{Case Description}

A 57-year-old man presented with uncontrolled intraocular pressure (IOP) and topical anti-glaucoma drug intolerance in his left eye. The patient's ocular history revealed that he had a history of PPV with SO tamponade and then intravitreal SO removal 5 years ago. Then,
${ }^{1-3}$ Department of Ophthalmology, Gazi University School of Medicine, Ankara, Turkey

Corresponding Author:Zeynep Aktas, Department of Ophthalmology, Gazi University School of Medicine, Ankara, Turkey, Phone: +903122026315, e-mail: drzeynep2000@yahoo.com

How to cite this article: Aktas Z, Bölük CE, Gurelik G. Silicone Oil Droplets in the Schlemm's Canal: A Surprise during Prolene Hemigonioscopy-assisted Transluminal Trabeculotomy (Hemi-GATT). J Curr Glaucoma Pract 2021;15(1):40-43.

Source of support: Nil

Conflict of interest: None

he had Ahmed glaucoma valve (AGV) implantation surgery for the treatment of secondary glaucoma developing 3 years after the SO removal. However, he was still on one anti-glaucoma medication. BCVAs were 20/30 and 20/200; IOPs were measured as 14 and $17 \mathrm{~mm}$ $\mathrm{Hg}$; in OD and OS, respectively. Both eyes were pseudophakic and the tube implant was in the anterior chamber (AC) with its normal position. On fundoscopic examination, the retina was attached in both eyes and he had 0.3 and 0.7 cup-to-disc ratios in both eyes, respectively.

Gonioscopic examination revealed Shaffer grade III angle and no synechia in the inferior quadrant. He was intolerant to all classes of medications including prostaglandins, beta-blockers, and fixed combinations for a long time, so surgical treatment was planned. Since he had no peripheral anterior synechia inferiorly, inferior hemi-GATT surgery was performed as an addition to AGV. Surgical steps were as follows: 
After a standard sterile preparation of the patient, the eye was draped and a lid speculum was placed. Temporal and superior paracentesis was placed using a 23-G microsurgical blade. A viscoelastic material (sodium hyaluronate) was injected into the AC. The microscope and the patient's head were then oriented to visualize the gonioscopic structures under the view of the Swan-Jacob goniolens. A 1-2 mm goniotomy was created using a microvitreoretinal (MVR) blade through the temporal site paracentesis. Right after the goniotomy, a bolus of emulsified SO droplets came out of the SC (Fig. 1). 6-0 prolene suture with a blunted tip by the hand-held cautery was introduced into AC through the superior quadrant paracentesis toward the angle. Microvitreoretinal forceps are then introduced through the same temporal site and the blunted tip of the 6-0 prolene suture was grasped with MVR forceps and then introduced into the SC. The suture was advanced in the canal inferiorly using the MVR forceps. Then, proximal part of the suture was pulled and inferior hemi-GATT was completed. Then, $A C$ was irrigated very well to wash the reflux bleeding and SO droplets spilled into the AC from the SC.

At the 2nd month follow-up, IOP was under control and measured as $10 \mathrm{~mm} \mathrm{Hg}$ without any medication (Fig. 2). Trabeculotomy area was noted inferiorly and nasally both with gonioscopy and anterior segment optical coherence tomography (Fig. 3).

\section{Discussion}

Gonioscopy-assisted transluminal trabeculotomy surgery has been introduced to the ophthalmology literature in 2014 by Grover et al. ${ }^{7}$ It has been shown to reduce IOP successfully in cases with primary, juvenile, and secondary open-angle glaucomas. ${ }^{8,9}$ It has the advantage of being blebless surgery and also being an efficient angle procedure since it tears up the TM all the way around. It can also be performed in cases with a history of incisional glaucoma surgeries.

In cases with a history of previous PPV with SO tamponade and then SO removal; glaucoma might occur secondary to the presence of emulsified SO droplets. Normally the rates of glaucoma following uncomplicated PPV and intravitreal SO injection could be as high as $56 \%$ at 8 months; however, recent studies started to report lower rates and it might be the result of the improvements of the vitreoretinal surgical techniques. ${ }^{10}$ Higher viscosity SO has been reported to cause fewer SO droplets and secondary glaucomas. ${ }^{11}$

This type of glaucoma has been attributed to trabecular inflammation or emulsified SO droplets in the TM. This can develop in secondary open or closed angles after the PPV. ${ }^{12,13}$

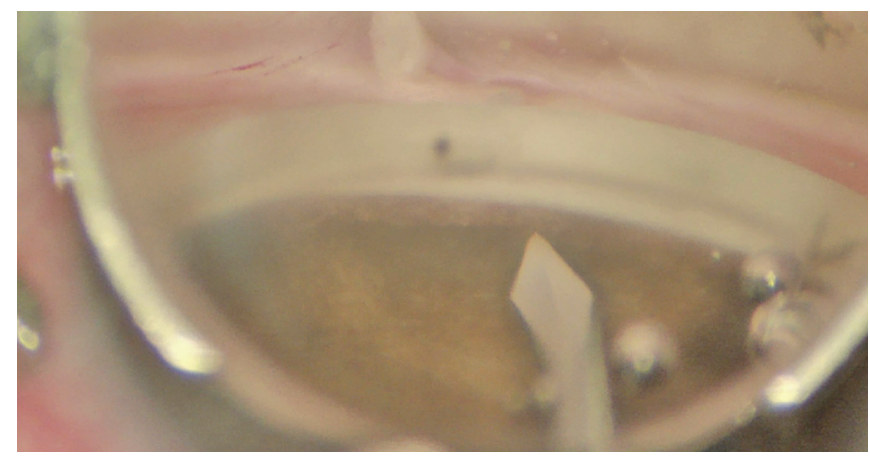

Fig. 1: Silicone oil droplets coming out of the Schlemm's canal after the goniotomy
Quan et al. ${ }^{6}$ have recently reported the results of GATT surgery in patients with secondary open-angle glaucoma after vitreoretinal surgery. In this study, they retrospectively evaluated the results of eight patients, in which only five of them underwent PPV with SO tamponade and all of them had already SO in the vitreous cavity at the time of GATT surgery.

In these cases with glaucoma secondary to SO droplets after PPV and SO removal, if medical treatment fails; filtering and tube surgery may be indicated as well. However, in those with a history of PPV and conjunctival scarring, tube surgery might be a better option.

This case was intolerant to all classes of anti-glaucoma medications throughout his follow-up. He first had tube surgery for the treatment of emulsified SO droplet glaucoma but unfortunately, he needed one anti-glaucoma medication to reach the target IOP 4 months after the tube surgery. IOP was 17 with AGV plus one medication. However, he had severe allergic reactions to all classes of medications and intolerance again. Thus, GATT surgery was planned as an adjunct to AGV to have the patient off medication. Since the IOP was already controlled with medication, inferior hemi-GATT $\left(180^{\circ}\right)$ was performed instead of conventional GATT surgery. Hemi-gonioscopy-assisted transluminal trabeculotomy might also have the advantage of causing less hyphema because of its less invasive nature compared to $360^{\circ}$ GATT according to our clinical observations.

Interestingly, during the surgery, it was noted that SC was full of emulsified SO droplets spilled into the AC right after the goniotomy. Madanagopalan et al. ${ }^{14}$ showed emulsified SO droplets in the SC with AS-OCT in a 55-year-old male patient with emulsified SO completely filling the AC. On the other hand, SO droplets might also transfer through the sclerotomy site or tube. ${ }^{15,16}$ Silicone oil removal and its role in reducing IOP is controversial. Its success rate on IOP reduction ranges from 0 to $93.4 \%$ in the current literature. ${ }^{17}$ Despite multiple attempts of air-fluid exchange for the removal of $\mathrm{SO}$, it is hard to get rid of all SO droplets spilled into the eye. In the current case, despite he had a history of SO removal, he still had SO droplets in the eye; but mostly in the SC while almost none in the AC. We noticed that SC was full of emulsified SO droplets right after the goniotomy incision. Thus, this might explain why IOP might be resistant that much even after the SO removal in these eyes.

To the best of our knowledge, our case is the first one that emulsified SO droplets have been shown during GATT surgery.

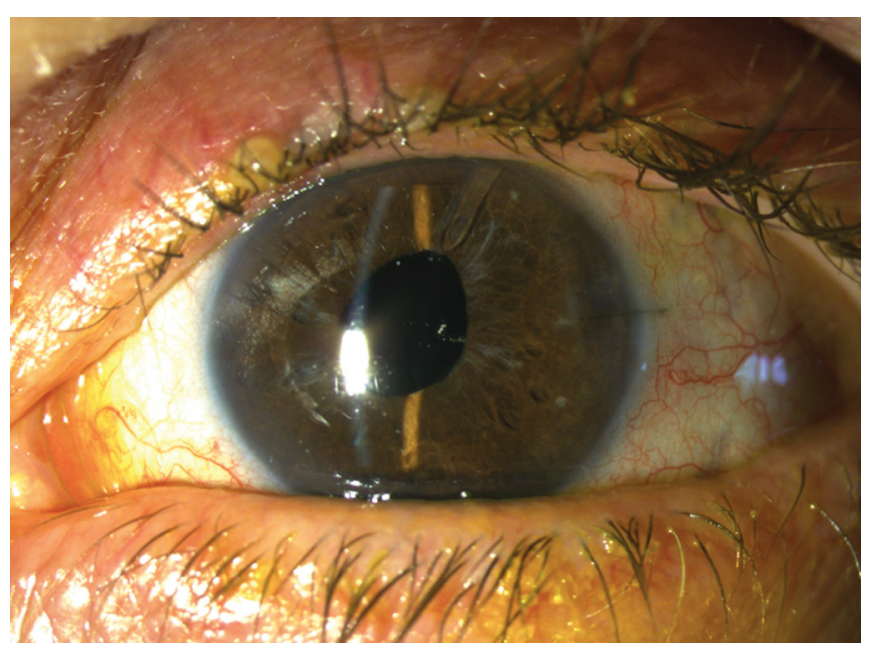

Fig. 2: Postoperative appearance of the eye 2 months after the surgery 

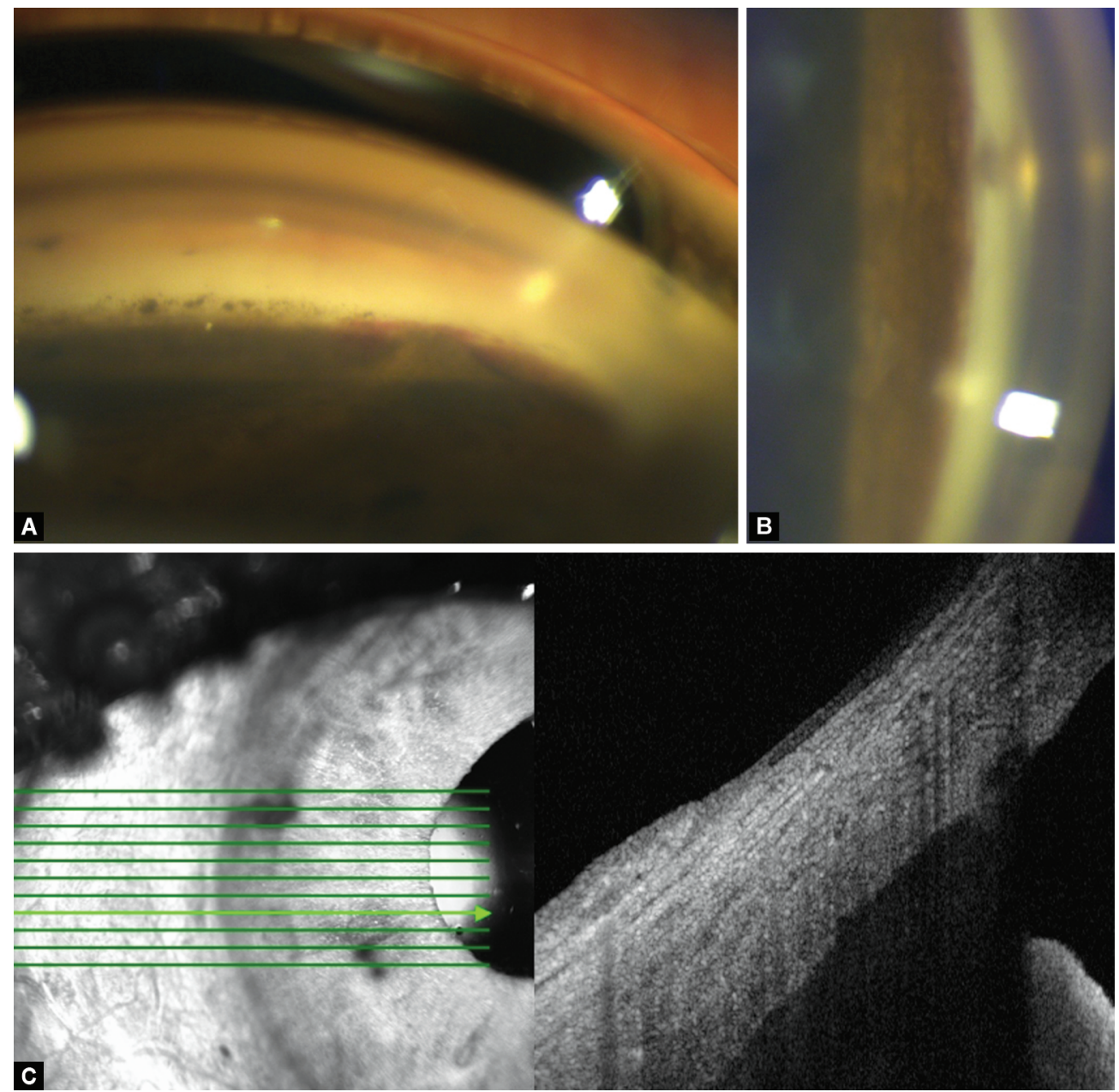

Figs 3A to C: Inferior angle, peripheral anterior synechia, and blood reflux in the Schlemm's canal and pigments over the angle (A); nasal angle, white posterior wall of the Schlemm's canal (B); Anterior segment optical coherence tomography showing trabeculotomy area (C); 2 months after the surgery

Thus, in these eyes with a history of PPV-SO removal and secondary glaucoma, the angle should be checked. If the angle is open GATT or hemi-GATT surgery as a primary or an adjunctive surgery makes sense in these cases since the primary mechanism might be TM dysfunction secondary to emulsified SO droplets or their presence in the SC as we have reported here. On the other hand, GATT surgery brings the advantages of its minimally invasive nature, which is very important in patients with scarred conjunctiva due to previous PPV and SO removal surgeries.

\section{Conclusion}

The presence of SO droplets in the SC might contribute to the development of secondary glaucoma after PPV and SO removal. Gonioscopy-assisted transluminal trabeculotomy or hemi-GATT surgery might be safe and effective surgical techniques in secondary open-angle glaucoma cases with a previous history of PPV and SO removal. Future prospective and randomized studies are needed to demonstrate its success in these cases.

\section{Clinical Significance}

Silicone oil droplets might be seen in the SC and contribute to the development of glaucoma after PPV and SO removal.
Gonioscopy-assisted transluminal trabeculotomy might be the less invasive and targeted surgical treatment method for the treatment of these cases.

\section{References}

1. Chang S. LXII Edward Jaackson lecture: open angle glaucoma after vitrectomy. Am J Ophthalmol 2006;141(6):1033-1043. DOI: 10.1016/j. ajo.2006.02.014.

2. Koreen L, Yoshida N, Escariao P, et al. Incidence of, risk factors for, and combined mechanism of late-onset open-angle glaucoma after vitrectomy. Retina 2012;32(1):160-167. DOI: 10.1097/ IAE.0b013e318217fffb.

3. Alkin Z, Satana B, Ozkaya A, et al. Selective laser trabeculoplasty for glaucoma secondary to emulsified silicone oil after pars plana vitrectomy: a pilot study. Biomed Res Int 2014;2014:469163. DOI: 10.1155/2014/469163.

4. Honavar SG, Goyal M, Majji AB, et al. Glaucoma after pars plana vitrectomy and silicone oil injection for complicated retinal detachments. Ophthalmology 1999;106(1):169-176. DOI: 10.1016/ S0161-6420(99)90017-9.

5. Cardascia N, Cantatore F, Ferreri $P$, et al. Ex-press mini-implant in the management of ocular hypertension secondary to silicone oil tamponed. Med Hypothesis Discov Innov Ophthalmol 2016;5(1): $10-14$. 
6. Quan AV, Yannuzzi NA, Chen J, et al. Gonioscopy-assisted transluminal trabeculotomy (GATT) in patients with secondary open-angle glaucoma following vitreoretinal surgery. J Glaucoma 2020(4):e23e25. DOI: $10.1097 /$ IJG.0000000000001461.

7. Grover DS, Godfrey DG, Smith O, et al. Gonioscopy-assisted transluminal trabeculotomy, ab interno trabeculotomy: technique report and preliminary results. Ophthalmology 2014;121(4):855-861. DOI: 10.1016/j.ophtha.2013.11.001.

8. Grover DS, Fellman RL. Gonioscopy assisted transluminal trabeculotomy (GATT) for the treatment of adult and developmental glaucomas. In: Aref AA, Varma R, ed. Advanced glaucoma surgery. Switzerland: Springer International Publishing; 2015. pp. 41-50.

9. Grover DS, Smith O, Fellman RL, et al. Gonioscopy assisted transluminal trabeculotomy: an ab interno circumferential trabeculotomy for the treatment of primary congenital glaucoma and juvenile open angle glaucoma. Br J Ophthalmol 2015;99(8):1-5. DOI: 10.1136/bjophthalmol-2014-306269.

10. Al-Jazzaf AM, Netland PA, Charles S. Incidence and management of elevated intraocular pressure after silicone oil injection. J Glaucoma 2005;14(1):40-46. DOI: 10.1097/01.ijg.0000145811. 62095.fa.
11. Petersen J, Ritzau-Tondrow U. Chronic glaucoma following silicone oil implantation: a comparison of 2 oils of differing viscosity. Fortschr Ophthalmol 1988;85(6):632-634.

12. Ichhpujani P, Jindal A, Jay KL. Silicone oil induced glaucoma: a review. Graefes Arch Clin Exp Ophthalmol Albrecht Von Graefes Arch Klin Exp Ophthalmol 2009;247(12):1585-1593. DOI: 10.1007/s00417-009-1155-x.

13. Valone J, McCarthy M. Emulsified anterior chamber silicone oil and glaucoma. Ophthalmology 1994;101(12):1908-1912. DOI: 10.1016/ s0161-6420(94)31084-0.

14. Madanagopalan VG, Velis G, Devulapally S. Emulsified silicone oil droplets in the canal of Schlemm. Int Ophthalmol 2019;39(4):925-926. DOI: 10.1007/s10792-018-0877-3.

15. Senn P, Büchi ER, Daicker B, et al. Bubbles in the bleb: troubles in the bleb? Molteno implant and intraocular tamponade with silicone oil in an aphakic patient. Ophthalmic Surg 1994;25(6):379-382.

16. Hyung SM, Min JP. Subconjunctival silicone oil drainage through the Molteno implant. Korean J Ophthalmology 1998;12(1):73-75. DOI: 10.3341/kjo.1998.12.1.73.

17. Flaxel CJ, Mithchell SM, Aylward GW. Visual outcome after silicone oil removal and recurrent retinal detachment repair. Eye (Lond) 2000;14(Pt 6):834-838. DOI: 10.1038/eye.2000.232. 